\title{
Automated determination of the ankle-brachial index using an oscillometric blood pressure monitor: validation vs. Doppler measurement and cardiovascular risk factor profile
}

\begin{abstract}
Anastasios Kollias, Apostolos Xilomenos, Athanase Protogerou, Evangelos Dimakakos and George S Stergiou
The ankle-brachial index (ABI) is a method used widely for peripheral arterial disease (PAD) diagnosis and cardiovascular risk prediction. This study validated automated $\mathrm{ABI}$ measurements taken using an oscillometric blood pressure (BP) monitor allowing simultaneous arm-leg BP measurements. A total of 93 patients (hypertension $83 \%$; dyslipidemia $72 \%$; diabetes $45 \%$; cardiovascular disease $23 \%$; smoking $15 \%$ ) were submitted to Doppler and automated $A B I$ measurements, performed using a professional oscillometric BP monitor (Microlife WatchBP Office; triplicate simultaneous arm-leg BP measurements), in a randomized order. The mean difference between the Doppler reading $(1.08 \pm 0.17)$ and $(1)$ the first oscillometric $A B I$ reading was $0.03 \pm 0.11$, (2) the average of two oscillometric readings was $0.02 \pm 0.10$ and (3) the average of three oscillometric readings was $0.02 \pm 0.09(P<0.01$ for all $)$. Strong correlations were found between oscillometric and Doppler ABI $(r 0.80$, 0.85 and 0.86 for single and average of two and three oscillometric readings, respectively; $P<0.001$ for all). Agreement between oscillometric and Doppler ABI in diagnosing PAD (Doppler $A B I<0.9$ ) was found in $95 \%$ of cases ( 6.79 ; agreement in diabetics: $94 \%, \kappa 0.79$ ). A receiver operating characteristic (ROC) curve revealed area under the curve at 0.98 , with a 0.97 oscillometric ABI cutoff for optimal sensitivity $(92 \%)$ and specificity $(92 \%)$ in diagnosing PAD. Average time for automated ABI measurement was 5.8 vs. $9.3 \mathrm{~min}$ for Doppler $(P<0.001)$. Doppler and oscillometric $A B I$ were associated and predicted (multivariate regression analysis) by the same cardiovascular risk factors (pulse pressure, smoking and cardiovascular disease history). Automated ABI measurement using a professional BP monitor allowing simultaneous arm-leg BP measurements appears to be a reliable and faster alternative to Doppler measurement.

Hypertension Research (2011) 34, 825-830; doi:10.1038/hr.2011.53; published online 19 May 2011
\end{abstract}

Keywords: ankle-brachial index; atherosclerosis; blood pressure; oscillometry; peripheral arterial disease

\section{INTRODUCTION}

Peripheral arterial disease (PAD) is a frequent manifestation of atherosclerosis, particularly in the elderly, in patients with diabetes and in those with multiple cardiovascular risk factors. ${ }^{1,2}$ Even in its asymptomatic form, the presence of $\mathrm{PAD}$ has been shown to be associated with an increased risk for cardiovascular morbidity and mortality. ${ }^{3}$ The Doppler-measured ankle-brachial index (ABI) is a relatively simple and noninvasive method for the assessment of PAD and the prediction of cardiovascular risk. ${ }^{1,4-6}$ Current guidelines provided by the European Society of Hypertension-European Society of Cardiology endorse the ABI measurement as a 'recommended' test in hypertensive patients, with values $<0.9$ indicating advanced atherosclerosis and increased cardiovascular risk. ${ }^{7}$ However, its clinical application is limited by the need for specialized equipment and the time required for measurement, which leads to underdiagnosis of asymptomatic PAD. ${ }^{8,9}$
Oscillometric determination of blood pressure (BP) has emerged as a simple, accurate and widely available technique for measurement in the doctor's office, at home or with ambulatory monitoring. ${ }^{10}$ Recent studies have shown that automated determination of ABI using oscillometric BP monitors appears to be a useful alternative to the conventional manual measurement by Doppler. ${ }^{11-14}$ However, there is evidence that in diabetic patients, who often have underdiagnosed PAD, oscillometric ABI does not correlate as closely with Doppler ABI as in non-diabetics. ${ }^{12}$ In addition, oscillometric ABI values have not been validated in terms of their clinical relevance.

This study was designed to validate automated ABI measurement using a professional oscillometric BP monitor that allows simultaneous arm-leg BP measurements. We compared these results with those obtained using the reference manual method with Doppler in diabetic and non-diabetic patients. The validation process consisted of two parts: (1) measurement validation, which compared Doppler and 
oscillometric ABI values and assessed their association; and (2) clinical validation, which compared the diagnosis of PAD by the two methods and assessed the association of automated and Doppler ABI values with cardiovascular risk factors.

\section{METHODS}

\section{Study population}

Patients with various cardiovascular risk factors attending a hypertension or a diabetes outpatient clinic were invited to participate in the study. Subjects with atrial fibrillation, incompressible ankle arteries $(\mathrm{ABI} \geqslant 1.4)$, excessive ankle edema or inflammatory ankle lesions were excluded. The study protocol was approved by the hospital scientific committee, and all participants provided signed informed consent.

\section{Blood pressure and $\mathrm{ABI}$ measurements}

Before initiation of the study, two observers made automated oscillometric and manual (Doppler) ABI measurements in 11 patients to gain familiarity with the test device. Both observers fulfilled the British Hypertension Society Protocol criteria for their agreement in simultaneous BP measurement ( 5 subjects, total of 50 measurements) and also performed simultaneous arm systolic BP measurements with the Doppler device in 4 subjects (total of 40 measurements). ${ }^{15}$

The study protocol included three steps: (1) an introductory familiarization with automated ABI measurement; (2) three simultaneous oscillometric BP measurements of both arms (in case of a consistent interarm difference of $\geqslant 12 \mathrm{~mm} \mathrm{Hg}$, the arm with the higher BP was selected for the subsequent oscillometric ABI measurements; otherwise, the right arm was used); and (3) Doppler and automated ABI measurement in randomized order (Doppler ABI was measured once, whereas automated ABI calculation included triplicate simultaneous arm-leg measurements, performed for each side at 30-s intervals). The occurrence of five sequential oscillometric errors was defined as a failure of the oscillometric device to measure ABI.

All study measurements were performed under standardized conditions in a quiet examination room with 10-min rest in the supine position before the introductory familiarization measurement. The manual Doppler and automated oscillometric ABI measurements were performed by a single investigator. Manual Doppler ABI was measured according to the American Heart Association guidelines using a continuous wave Doppler device (Hadeco Bidop ES-100V3, Kawasaki, Japan) with an $8 \mathrm{MHz}$ probe. ${ }^{1}$ Systolic BP was defined with a standard mercury sphygmomanometer by the first Doppler flow signal while deflating the cuff from a suprasystolic level in brachial, dorsalis pedis and posterior tibial arteries. BP cuffs were placed on the arm (with the lower edge $\sim 1$ inch above the antecubital fossa; cuff placed on the brachial artery) and on the lower calf $(\sim 1-2$ inches above the ankle's medial malleolus; conical cuff placed on the posterior tibial artery). Systolic pressure was determined sequentially for the brachial, dorsalis pedis and posterior tibial arteries for each side, and ABI was calculated for each leg by dividing the highest ankle pressure by the highest arm pressure. ${ }^{1}$ Automated ABI measurement was performed using a validated oscillometric BP monitor designed for professional use in the office (WatchBP Office device, Microlife, Widnau, Switzerland). ${ }^{16}$ This device allows automated simultaneous BP measurements of both arms or one arm and one leg, and thereby the calculation of ABI. The ABI results are displayed on the device screen.

Doppler and oscillometric measurements were performed using the same cuffs (different for arm and ankle), with bladder cuff size selected according to the circumference of the arm or leg (inflatable bladder size $23.5 \times 13 \mathrm{~cm}$ and $29 \times 15 \mathrm{~cm}$ for arm cuff and $23 \times 14 \mathrm{~cm}$ and $29 \times 15 \mathrm{~cm}$ for leg cuff).

\section{Statistical analysis}

The inter- and intra-observer variabilities of Doppler systolic BP measurements were assessed by determination of the intraclass correlation coefficient of agreement. A paired $t$-test was applied for the comparison of ABI values measured using different methods in the same subjects, and the Wilcoxon test was used to compare the time needed for $\mathrm{ABI}$ measurement with the two methods. The level of agreement between Doppler and oscillometric ABI values was assessed by (1) Pearson's correlation coefficients, (2) s.d. of differences and Bland-Altman scatter plots, (3) $\kappa$ statistic for agreement in PAD diagnosis, defined as Doppler ABI $<0.9$ and (4) receiver operator characteristic (ROC) curves in the whole sample as well as in the diabetic and non-diabetic subgroups. The $z$-statistic was performed for the comparison of correlation coefficients as well as of ROC curves. Stepwise multiple regression analysis was applied to identify predictors of ABI values and of Doppler-oscillometric ABI differences. The results are expressed as the mean values with s.d. A probability value of $P<0.05$ was considered statistically significant. Statistical analysis was performed using the Statistical Package for Social Sciences software (SPSS release 13.0; SPSS, Chicago, IL, USA).

\section{RESULTS}

\section{Participant characteristics}

A total of 98 subjects were invited to participate. Two subjects were excluded because of atrial fibrillation, and three refused to participate. Thus, 93 subjects were included in the analysis. The main characteristics of the study participants are presented in Table 1.

\section{Inter- and intra-observer variability}

The intraclass correlation coefficient of agreement between the two observers in performing 40 simultaneous systolic BP measurements of the arm with the Doppler device was 0.98 . The intraclass correlation coefficient of agreement between two measurements on the same subjects by the observer who performed all the ABI measurements was 0.92 .

\section{Validation of ABI measurement}

Mean Doppler ABI (average of ABI values per leg) was slightly lower than the first oscillometric ABI measurement (1.08 \pm 0.17 and $1.11 \pm 0.17$, respectively (mean difference $0.03 \pm 0.11,95 \%$ confidence interval $0.01-0.05, P<0.001))$. In addition, 27 legs $(27 / 186=15 \%, 16$ subjects) had a Doppler ABI of $<0.90$, which is used as the cutoff value to diagnose PAD. ${ }^{1,7}$ Pedal pulses were not palpable in 15 of these 27 limbs.

Valid oscillometric ABI measurements were obtained in 183 legs (98.4\%), whereas in 3 legs $(1.6 \%, 3$ patients), the device failed to measure ABI (all had Doppler ABI <0.90). The frequency of errors with the oscillometric method was higher in limbs with PAD compared with those without PAD (35.2 vs. 5.7\%, respectively; $P<0.001$ ). Among limbs with Doppler ABI $<0.90$, there was a tendency for a higher frequency of errors in those with nonpalpable ankle pulses

\section{Table 1 Participants' characteristics}

\begin{tabular}{lc}
\hline Males & $58(62)$ \\
Age (years) & $62.5 \pm 11.1$ \\
Waist circumference (cm) & $103.9 \pm 10$ \\
Office systolic BP (mm Hg) & $136.6 \pm 15.3$ \\
Office diastolic BP (mm Hg) & $78.8 \pm 9$ \\
Hypertension & $77(83)$ \\
Diabetes & $42(45)$ \\
Dyslipidemia & $64(72)$ \\
Current smokers & $14(15)$ \\
Past smokers & $36(39)$ \\
Cardiovascular disease & $21(23)$ \\
Chronic renal disease & $6(7)$ \\
Treatment with $\beta$-blockers & $20(22)$ \\
Limbs with Doppler ABI 0.6-0.9 & $20(11)$ \\
Limbs with Doppler ABI <0.6 & $7(4)$
\end{tabular}

Abbreviations: $\mathrm{ABI}$, ankle-brachial index; $\mathrm{BP}$, blood pressure. $N=93$, mean values \pm s.d., percent in parentheses. 

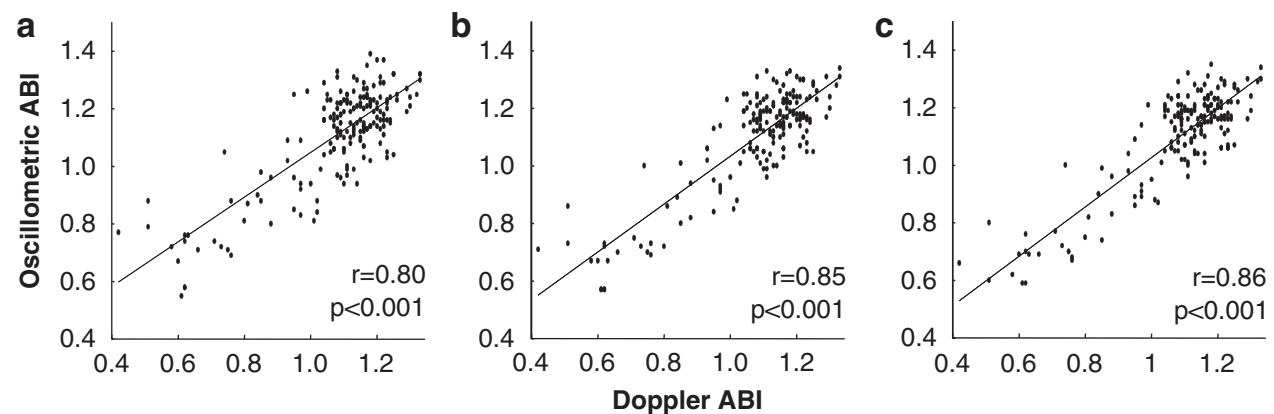

Figure 1 Correlation between Doppler and oscillometric ankle-brachial index (ABI) values when the first oscillometric measurement (a) or the mean of two (b) or three (c) oscillometric measurements was used.
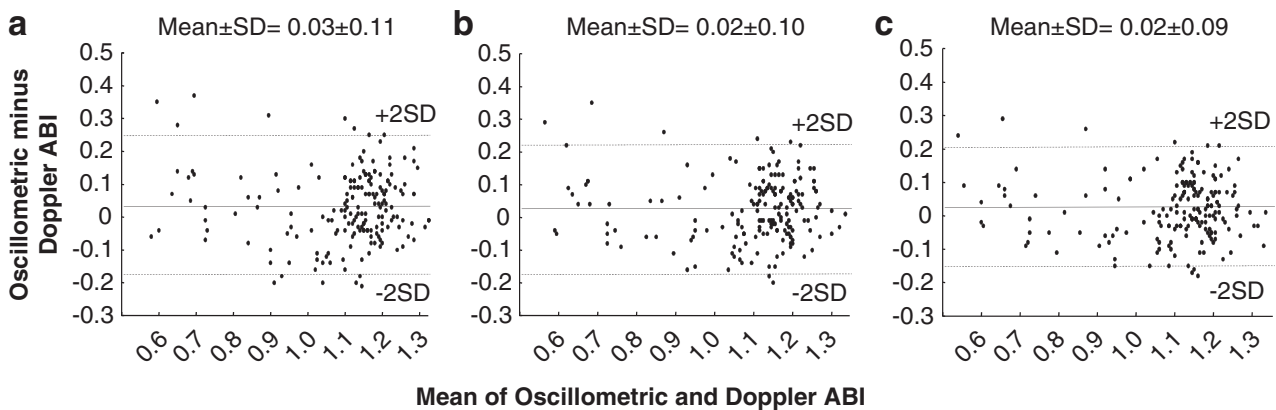

Figure 2 Bland-Altman scatter plots for the difference between Doppler and oscillometric ankle-brachial index (ABI) values when only the first oscillometric measurement (a) or the mean of two (b) or three (c) oscillometric measurements was used.

compared with those with palpable ankle pulses (40.3 vs. $15.2 \%$, respectively; $P=0.07)$.

Oscillometric ABI values were closely associated with Doppler ABI with slightly higher correlation coefficients when the mean of two or three oscillometric measurements was used in the analysis (Figure 1). Furthermore, there was a slight decrease in the mean Doppleroscillometric ABI difference and its s.d. when averaging two or three oscillometric readings compared with using the first reading only (Figure 2). In the stepwise multivariate regression analysis with age, gender, pulse pressure, lipid values (total cholesterol, low-density and high-density lipoprotein cholesterol, triglycerides), presence of diabetes, smoking status, presence of chronic renal failure, history of cardiovascular disease, treatment with $\beta$-blockers and presence of PAD as independent variables, only the presence of PAD could be identified as an independent predictor of the difference between the oscillometric (first reading) $\mathrm{ABI}$ and Doppler $\mathrm{ABI}(\beta \pm$ s.e. $=0.07 \pm 0.03)$. The difference between the Doppler ABI and the oscillometric ABI (mean \pm s.d.) was $0.09 \pm 0.13$ in limbs with $\operatorname{PAD}(P=0.002)$ and $0.02 \pm 0.10(P=0.01)$ in those without PAD.

When excluding the 20 subjects receiving $\beta$-blockers, there was no difference in the results: mean oscillometric-Doppler ABI difference: $0.03 \pm 0.11, P=0.001$; correlation coefficient between oscillometric and Doppler ABI: $0.79 ; P<0.001$.

The average time required for $\mathrm{ABI}$ measurement (excluding time needed for the initial placement of the cuffs, which was common for both methods and was performed during the initial 10-min rest period) was longer using the manual Doppler method $(9.3 \pm$ $2.2 \mathrm{~min}$, single measurement) compared with the automated oscillometric method ( $5.8 \pm 0.3 \mathrm{~min}$ for three simultaneous measurements in both arms plus two simultaneous arm-leg measurements, with a 30-s interval between readings) $(P<0.001)$.

\section{Clinical validation of $A B I$}

PAD diagnosis. The agreement between the Doppler and oscillometric (first reading) methods in diagnosing PAD was 95\% ( $\kappa 0.79$ ). By using the 0.90 cutoff, the oscillometric ABI measurement had a sensitivity of $83 \%$ and a specificity of $97 \%$ in diagnosing PAD. When the ROC curve was computed for oscillometric ABI (Figure 3), the area under the curve was 0.981 and the oscillometric $A B I$ cutoff value for optimal sensitivity and specificity (both at 92\%) in diagnosing PAD was 0.97 .

Association with cardiovascular risk factors. The bivariate correlations between $\mathrm{ABI}$ values and cardiovascular risk factors are shown in Table 2. No significant difference was observed between the coefficients of oscillometric (first reading) or Doppler ABI and cardiovascular risk factors. In stepwise multivariate regression analysis with age, gender, pulse pressure, total cholesterol, low-density lipoprotein and high-density lipoprotein cholesterol, triglycerides, presence of diabetes, smoking status, presence of chronic renal disease, history of cardiovascular disease and treatment with $\beta$-blockers as independent variables, Doppler ABI was best predicted $\left(R^{2}=0.26\right)$ by pulse pressure $(\beta \pm$ s.e. $=-0.005 \pm 0.001)$, smoking status $(-0.06 \pm$ $0.02)$ and history of cardiovascular disease $(-0.09 \pm 0.04)$, whereas oscillometric $\mathrm{ABI}$ was best determined $\left(R^{2}=0.31\right)$ by pulse pressure $(-0.004 \pm 0.001)$, smoking status $(-0.06 \pm 0.02)$, history of cardiovascular disease $(-0.08 \pm 0.04)$ and low-density lipoprotein cholesterol $(-0.001 \pm 0.001)$.

Diabetic vs. non-diabetic subjects. The mean ABI tended to be lower in diabetic subjects $(n=42)$ compared with non-diabetic subjects $(n=51)$ using either the Doppler $(1.05 \pm 0.22$ and $1.09 \pm 0.15$, respectively; $P=0.16)$ or the automated oscillometric method $(1.09 \pm 0.18$ 


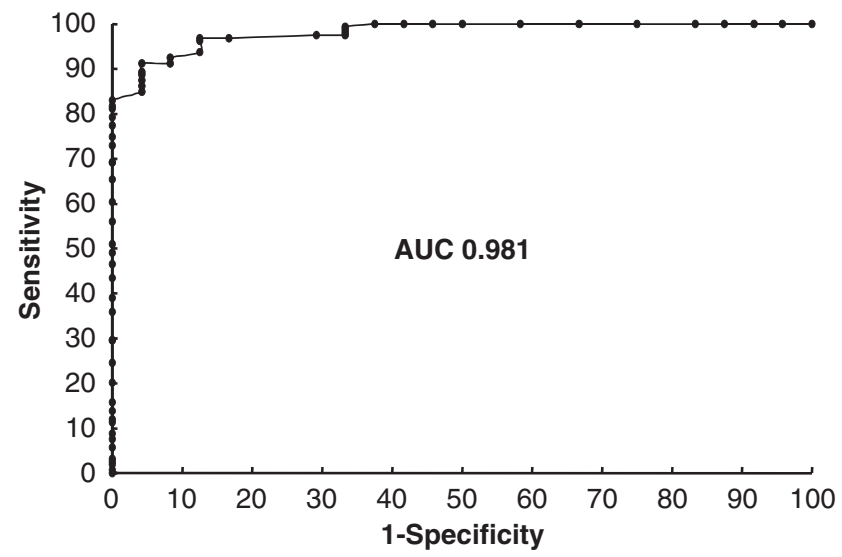

Figure 3 Receiver operating characteristic (ROC) curve for oscillometric ankle-brachial index $(A B I)$ in diagnosing peripheral arterial disease (PAD) as defined by Doppler $\mathrm{ABI}<0.90$.

and $1.13 \pm 0.16$, respectively; $P=0.18)$. The mean difference between the Doppler and the automated (first measurement) ABI measurements was similar in diabetics and non-diabetics $(0.03 \pm 0.12$ and $0.03 \pm 0.10$, respectively). The strength of the association between Doppler and oscillometric ABI was similar in non-diabetic $(r 0.80$, 0.83 and 0.84 for single and average of two and three oscillometric readings, respectively; $P<0.001$ for all) and diabetic subjects $(r 0.81$, 0.86 and 0.88 , respectively; $P<0.001$ for all). There was agreement between the Doppler and the oscillometric method in diagnosing PAD in $96 \%$ of non-diabetic subjects ( $\kappa$ 0.78) and in $94 \%$ of diabetics ( $\kappa$ 0.79). The ROC curve revealed area under the curve at 0.987 , with a 0.98 oscillometric ABI cutoff for optimal sensitivity and specificity (both at 93\%) in diagnosing PAD in non-diabetic subjects, whereas the respective values for diabetic patients were 0.973 (area under the curve) and 0.93 (cutoff for optimal sensitivity and specificity at 92\%). There was no significant difference between the ROC curves for diabetics $v s$. non-diabetics $(P=0.49)$.

\section{DISCUSSION}

This study validated automated ABI measurements taken by a validated professional oscillometric BP monitor, which allows simultaneous arm-leg BP measurements, compared with the reference Doppler method, which does not. The main findings were: (1) automated oscillometric ABI measurement was feasible in most patients, (2) Doppler and oscillometric ABI values were highly correlated, (3) the diagnostic ability of the oscillometric ABI measurement was very close to that of the Doppler method, (4) both Doppler and oscillometric ABI values were correlated with cardiovascular risk factors and shared almost the same predictors and (5) the results did not differ in diabetics when compared with non-diabetic subjects.

Automated oscillometric ABI measurement had broad applicability in this study, as it was feasible in $98 \%$ of cases. It should be noted that the study participants represent a typical population with clusters of cardiovascular risk factors seen in an outpatient cardiovascular risk factor clinic. Moreover, oscillometric measurements did not require special training and were less time consuming than the Doppler method. This was mainly attributed to the ability of the oscillometric device to allow simultaneous BP measurements of both arms or one arm and one leg. In real-world conditions, comparison in terms of time would require one measurement in each arm and each leg (using the dorsalis pedis for the Doppler). Even in that case, however, the time needed for Doppler measurement would be greater compared
Table 2 Bivariate correlation coefficients of Doppler and oscillometric $A B I$ values with cardiovascular risk factors

\begin{tabular}{lccc}
\hline Variables & Doppler ABI & Oscillometric ABI & P for difference \\
\hline Age & $-0.26^{*}$ & -0.19 & $\mathrm{NS}$ \\
Waist circumference & -0.09 & $-0.33^{*}$ & $\mathrm{NS}$ \\
Systolic BP & $-0.28^{*}$ & $-0.25^{*}$ & $\mathrm{NS}$ \\
Diastolic BP & 0.11 & 0.16 & $\mathrm{NS}$ \\
Pulse pressure & $-0.41^{*}$ & $-0.40^{*}$ & $\mathrm{NS}$ \\
Total cholesterol & -0.08 & -0.09 & $\mathrm{NS}$ \\
LDL cholesterol & -0.06 & -0.13 & $\mathrm{NS}$ \\
HDL cholesterol & 0.09 & 0.16 & $\mathrm{NS}$ \\
Triglycerides & -0.14 & -0.20 & $\mathrm{NS}$ \\
Smoking status & $-0.27^{*}$ & $-0.30^{*}$ & $\mathrm{NS}$ \\
\hline
\end{tabular}

Abbreviations: $\mathrm{ABI}$, ankle-brachial index; $\mathrm{BP}$, blood pressure; $\mathrm{HDL}$, high-density lipoprotein; LDL, low-density lipoprotein; NS, not significant. ${ }^{*} P<0.05$

with that for the oscillometric one because of the necessity of additional steps such as pulse palpation, application of gel and signal viewing. Additionally, automated calculation of the ABI with the oscillometric device prevents calculation errors by the observer and saves time. Another advantage of the automated simultaneous oscillometric $\mathrm{ABI}$ measurement is that it is free of the random $\mathrm{BP}$ variation that occurs with sequential Doppler ABI measurement. Moreover, the automated method is free of interobserver variability and observer bias, which are additional problems characteristic of Doppler ABI measurement.

In this study, oscillometric ABIs were highly correlated with Doppler ABI values. As this correlation tended to be only marginally stronger when the mean of two or three oscillometric measurements was included in the analysis, a single measurement seems to be adequate for automated ABI assessment in clinical practice. Previous studies have reported various results concerning the correlation between oscillometric and Doppler ABI measurements. In particular, some studies revealed only a weak relationship between the oscillometric and Doppler methods, especially in patients with PAD. ${ }^{17-19} \mathrm{~A}$ modest agreement was also recently revealed between the oscillometric and Doppler technique in 146 subjects, with a correlation coefficient of 0.53. ${ }^{12}$ However, Beckman et al., ${ }^{11}$ validating the oscillometric method in a population of 201 subjects, found a correlation coefficient of 0.78 in both legs, whereas Gomez-Huelgas et al. ${ }^{20}$ reported a coefficient of 0.86 . In other studies, the automated-manual ABI differences averaged $\sim 0.03$ units. ${ }^{13,14}$ Taking these studies into account, it can be suggested that the automated ABI values obtained in this study showed a high correlation with Doppler values, which is among the highest reported in the literature. It should be noted that in most of the aforementioned studies, the oscillometric devices used were not formally validated or were proven unreliable or questionable for BP measurement (http://www.dableducational.org).

Another interesting finding in this study is that the oscillometric ABI values were slightly higher compared with Doppler values, which was also reported by Beckman et al. ${ }^{11}$ From a cardiovascular physiology point of view, this is not justified, because BP is assessed at the lower limb more distally by the Doppler than by the oscillometric device. Hence, ABI as assessed by the Doppler should be higher because of higher amplification of systolic BP distally as a result of the existence of pressure wave reflections and an arterial stiffness gradient within the arterial tree. ${ }^{21,22}$ Therefore, the difference found between the oscillometric and Doppler methods cannot be attributed to the slightly different points of ankle BP measurement but rather to the 
characteristics of each method. However, taking BP some inches more proximally (closer to the knee joint) may fail to detect arterial occlusive disease present between the cuff and the two pedal pulses. ${ }^{11}$ A systematic error in assessing the Doppler ABI, likely because of human error (the time lag between hearing the Doppler signal, viewing it and then recording the pressure might differ for arm and ankle), cannot be excluded.

When agreement studies in the diagnosis of PAD were performed by applying criteria with high clinical relevance $(\kappa$ statistic, ROC curve), the oscillometric technique was found to be quite reliable. More specifically, the agreement between the two techniques was satisfactory, with high $\kappa$ values. Furthermore, when the value of 0.90 was used as a cutoff, the oscillometric method had a high specificity at the expense of sensitivity. To optimize specificity and sensitivity for the diagnosis of $\mathrm{PAD}$, the ROC curve was computed, showing that the highest sensitivity and specificity (>90\%) were achieved at a cutoff value of 0.97 . This finding is in agreement with previous studies reporting that the oscillometric cutoff point to define PAD would have to be increased from 0.90 to $\sim 1 .^{12,20}$ Furthermore, repeated oscillometric errors were observed more commonly in limbs with PAD. This observation is in line with data reported by Gomez-Huelgas et al. ${ }^{20}$ and suggests that the inability of the oscillometric method to measure $\mathrm{ABI}$ should be interpreted as an indication for the presence of PAD and the need for further imaging (ultrasound, angiography). Indeed, if the inability of the oscillometric method to measure ABI was considered as abnormal $\mathrm{ABI}$, then the agreement between the two methods would be increased ( $\kappa 0.81$ ).

The presence of PAD is two to four times more prevalent in diabetic patients than in the general population. ${ }^{23}$ Clairotte et al. ${ }^{12}$ recently validated oscillometric $\mathrm{ABI}$ measurement in diabetic patients and reported lower correlation coefficients $(r=0.49$ vs. 0.60$)$ and higher differences between the two methods $(0.03 \pm 0.25 v s .0 .01 \pm 0.28)$ in diabetics compared with non-diabetics. In contrast, the findings of this study did not differ with regard to the presence of diabetes, and the diagnostic performance of the oscillometric method was similar in diabetics and non-diabetics.

Beyond the assessment of measurement accuracy by comparison with the reference method (Doppler), an alternative approach for validation of the automated $\mathrm{ABI}$ is the assessment of its correlation with established cardiovascular risk factors. Indeed, both the Doppler and oscillometric ABI values showed similar correlations with cardiovascular risk factors and shared similar predictors in multivariate analyses. Cardiovascular risk factors, such as hypertension, dyslipidemia, diabetes and smoking, are established risk factors for the presence of PAD. ${ }^{1}$ The association of the automated oscillometric ABI with such risk factors is particularly reassuring for its clinical relevance.

It should be noted that some important hemodynamic factors related to cardiac and arterial properties and functions that might have affected the findings of this study have not been evaluated. In particular, medical treatment and left ventricular stroke volume might be confounding factors in such studies. ${ }^{24-26}$ Among drugs, $\beta$-blockers are important because they affect the rate at which the pressure wave develops and thus the rate of change in arterial wall expansion. However, the inclusion of treatment with $\beta$-blockers in the analyses did not affect the relationship of Doppler with oscillometric ABI values or their association with other clinical parameters.

The findings of this study should be interpreted in light of certain limitations. The $1.6 \%$ failure rate seems to be lower than that recorded by other studies. ${ }^{12,17,18,20}$ However, the percentage of erroneous measurements was higher, particularly among limbs with Doppler ABI $<0.90$ and nonpalpable ankle pulses. It should be noted that the population studied was seen mainly in primary care clinics and not in specialized surgery vascular laboratories, which means that the proportion of subjects with severe PAD was relatively low. Thus, the findings of the current study cannot be extrapolated to all settings, but they do validate the device for use for massive screening in primary care.

In conclusion, these data suggest that the automated ABI determination obtained by simultaneous arm-leg BP measurements taken using a validated oscillometric device is highly correlated with Doppler readings and provides similar information in both diabetic and nondiabetic subjects. Moreover, this technique does not require special training and is less time consuming and free of observer bias. These features make this technique particularly attractive in terms of clinical applicability in everyday practice.

\section{CONFLICT OF INTEREST}

GS Stergiou has received consultation fees and research support from Microlife, Widnau, Switzerland.

\section{ACKNOWLEDGEMENTS}

This study was partially supported by Microlife, Widnau, Switzerland, and by the Hypertension Center, Third University Department of Medicine, Sotiria Hospital, Athens, Greece.

1 Hirsch AT, Haskal ZJ, Hertzer NR, Bakal CW, Creager MA, Halperin JL, Hiratzka LF, Murphy WR, Olin JW, Puschett JB, Rosenfield KA, Sacks D, Stanley JC, Taylor Jr LM, White CJ, White J, White RA, Antman EM, Smith Jr SC, Adams CD, Anderson JL, Faxon DP, Fuster V, Gibbons RJ, Hunt SA, Jacobs AK, Nishimura R, Ornato JP, Page RL, Riegel $B$, American Association for Vascular Surgery; Society for Vascular Surgery; Society for Cardiovascular Angiography and Interventions; Society for Vascular Medicine and Biology; Society of Interventional Radiology; ACC/AHA Task Force on Practice Guidelines Writing Committee to Develop Guidelines for the Management of Patients With Peripheral Arterial Disease; American Association of Cardiovascular and Pulmonary Rehabilitation; National Heart, Lung, and Blood Institute; Society for Vascular Nursing; TransAtlantic Inter-Society Consensus; Vascular Disease Foundation. ACC/AHA 2005 Practice Guidelines for the management of patients with peripheral arterial disease (lower extremity, renal, mesenteric, and abdominal aortic): a collaborative report from the American Association for Vascular Surgery/Society for Vascular Surgery, Society for Cardiovascular Angiography and Interventions, Society for Vascular Medicine and Biology, Society of Interventional Radiology, and the ACC/AHA Task Force on Practice Guidelines (Writing Committee to Develop Guidelines for the Management of Patients With Peripheral Arterial Disease): endorsed by the American Association of Cardiovascular and Pulmonary Rehabilitation; National Heart, Lung, and Blood Institute; Society for Vascular Nursing; TransAtlantic Inter-Society Consensus; and Vascular Disease Foundation. Circulation 2006; 113: e463-e654.

2 Diehm C, Schuster A, Allenberg JR, Darius H, Haberl R, Lange S, Pittrow D, von Stritzky B, Tepohl G, Trampisch HJ. High prevalence of peripheral arterial disease and comorbidity in 6880 primary care patients: cross-sectional study. Atherosclerosis 2004; 172: 95-105.

3 Diehm C, Allenberg JR, Pittrow D, Mahn M, Tepohl G, Haberl RL, Darius H, Burghaus I, Trampisch HJ, German Epidemiological Trial on Ankle Brachial Index Study Group. Mortality and vascular morbidity in older adults with asymptomatic versus symptomatic peripheral artery disease. Circulation 2009; 120: 2053-2061.

4 Fowkes FG, Murray GD, Butcher I, Heald CL, Lee RJ, Chambless LE, Folsom AR, Hirsch AT, Dramaix M, deBacker G, Wautrecht JC, Kornitzer M, Newman AB, Cushman M, Sutton-Tyrrell K, Fowkes FG, Lee AJ, Price JF, d'Agostino RB, Murabito JM, Norman PE, Jamrozik K, Curb JD, Masaki KH, Rodríguez BL, Dekker JM, Bouter LM, Heine RJ, Nijpels G, Stehouwer CD, Ferrucci L, McDermott MM, Stoffers HE, Hooi JD, Knottnerus JA, Ogren M, Hedblad B, Witteman JC, Breteler MM, Hunink MG, Hofman A, Criqui $\mathrm{MH}$, Langer RD, Fronek A, Hiatt WR, Hamman R, Resnick HE, Guralnik J, McDermott MM. Ankle brachial index combined with Framingham Risk Score to predict cardiovascular events and mortality: a meta-analysis. JAMA 2008; 300: 197-208.

5 Lijmer JG, Hunink MG, van den Dungen JJ, Loonstra J, Smit AJ. ROC analysis of noninvasive tests for peripheral arterial disease. Ultrasound Med Biol 1996; 22 : 391-398.

6 Stoffers HE, Kester AD, Kaiser V, Rinkens PE, Kitslaar PJ, Knottnerus JA. The diagnostic value of the measurement of the ankle-brachial systolic pressure index in primary health care. J Clin Epidemiol 1996; 49: 1401-1405.

7 Mancia G, De Backer G, Dominiczak A, Cifkova R, Fagard R, Germano G, Grassi G, Heagerty AM, Kjeldsen SE, Laurent S, Narkiewicz K, Ruilope L, Rynkiewicz A, Schmieder RE, Boudier HA, Zanchetti A, Vahanian A, Camm J, De Caterina R, Dean V, Dickstein K, Filippatos G, Funck-Brentano C, Hellemans I, Kristensen SD, McGregor 
K, Sechtem U, Silber S, Tendera M, Widimsky P, Zamorano JL, Erdine S, Kiowski W, Agabiti-Rosei E, Ambrosioni E, Lindholm LH, Viigimaa M, Adamopoulos S, Agabiti-Rosei E, Ambrosioni E, Bertomeu V, Clement D, Erdine S, Farsang C, Gaita D, Lip G, Mallion JM, Manolis AJ, Nilsson PM, O'Brien E, Ponikowski P, Redon J, Ruschitzka F, Tamargo J, van Zwieten P, Waeber B, Williams B, Management of Arterial Hypertension of the European Society of Hypertension; European Society of Cardiology. 2007 Guidelines for the Management of Arterial Hypertension: The Task Force for the Management of Arterial Hypertension of the European Society of Hypertension (ESH) and of the European Society of Cardiology (ESC). J Hypertens 2007; 25: 1105-1187.

8 Hirsch AT, Criqui MH, Treat-Jacobson D, Regensteiner JG, Creager MA, Olin JW, Krook $\mathrm{SH}$, Hunninghake DB, Comerota AJ, Walsh ME, McDermott MM, Hiatt WR. Peripheral arterial disease detection, awareness, and treatment in primary care. JAMA 2001; 286: 1317-1324.

9 Belch JJ, Topol EJ, Agnelli G, Bertrand M, Califf RM, Clement DL, Creager MA, Easton JD, Gavin III JR, Greenland P, Hankey G, Hanrath P, Hirsch AT, Meyer J, Smith SC, Sullivan F, Weber MA, Prevention of Atherothrombotic Disease Network. Critical issues in peripheral arterial disease detection and management: a call to action. Arch Intern Med 2003; 163: 884-892.

10 O'Brien E, Asmar R, Beilin L, Imai Y, Mallion JM, Mancia G, Mengden T, Myers M, Padfield P, Palatini P, Parati G, Pickering T, Redon J, Staessen J, Stergiou G, Verdecchia P, European Society of Hypertension Working Group on Blood Pressure Monitoring. European Society of Hypertension recommendations for conventional, ambulatory and home blood pressure measurement. J Hypertens 2003; 21: 821-848.

11 Beckman JA, Higgins CO, Gerhard-Herman M. Automated oscillometric determination of the ankle-brachial index provides accuracy necessary for office practice. Hypertension 2006; 47: 35-38.

12 Clairotte C, Retout S, Potier L, Roussel R, Escoubet B. Automated ankle-brachial pressure index measurement by clinical staff for peripheral arterial disease diagnosis in non-diabetic and diabetic patients. Diabetes Care 2009; 32: 1231-1236.

13 Pan CR, Staessen JA, Li Y, Wang JG. Comparison of three measures of the ankle-brachial blood pressure index in a general population. Hypertens Res 2007; 30: 555-561.

14 Richart T, Kuznetsova T, Wizner B, Struijker-Boudier HA, Staessen JA. Validation of automated oscillometric versus manual measurement of the ankle-brachial index. Hypertens Res 2009; 32: 884-888.

15 O'Brien E, Petrie J, Littler W, de Swiet M, Padfield PL, Altman DG, Bland M, Coats A, Atkins N. An outline of the revised British Hypertension Society protocol for the evaluation of blood pressure measuring devices. J Hypertens 1993; 11: 677-679.
16 Stergiou GS, Tzamouranis D, Protogerou A, Nasothimiou E, Kapralos C. Validation of the Microlife Watch BP Office professional device for office blood pressure measurement according to the International protocol. Blood Press Monit 2008; 13: 299-303.

17 Salles-Cunha SX, Vincent DG, Towne JB, Bernhard VM. Noninvasive ankle pressure measurements by oscillometry. Tex Heart Inst J 1982; 9: 349-357.

18 Adiseshiah M, Cross FW, Belsham PA. Ankle blood pressure measured by automatic oscillotonometry: a comparison with Doppler pressure measurements. Ann R Coll Surg Engl 1987; 69: 271-273.

19 Ramanathan A, Conaghan PJ, Jenkinson AD, Bishop CR. Comparison of ankle-brachial pressure index measurements using an automated oscillometric device with the standard Doppler ultrasound technique. ANZ J Surg 2003; 73: 105-108.

20 Gomez-Huelgas R, Martinez-Gonzalez J, de Albornoz MC, Peña-Jiménez D, Sobrino-Díaz B, Bernal-López MR. Automated ankle-brachial pressure index measurement by clinical staff for peripheral arterial disease diagnosis in non-diabetic and diabetic patients: response to Clairotte et al. Diabetes Care 2010; 33: e41; author reply e42.

21 Avolio AP, Van Bortel LM, Boutouyrie P, Cockcroft JR, McEniery CM, Protogerou AD, Roman MJ, Safar ME, Segers P, Smulyan H. Role of pulse pressure amplification in arterial hypertension: experts' opinion and review of the data. Hypertension 2009; 54: 375-383.

22 Protogerou AD, Papadogiannis D, Safar ME. Letter by Protogerou et al. regarding article, "mortality and vascular morbidity in older adults with asymptomatic versus symptomatic peripheral artery disease". Circulation 2010; 121: e455.

23 Gregg EW, Sorlie P, Paulose-Ram R, Gu Q, Eberhardt MS, Wolz M, Burt V, Curtin L, Engelgau M, Geiss L, 1999-2000 national health and nutrition examination survey. Prevalence of lower-extremity disease in the US adult population $>=40$ years of age with and without diabetes: 1999-2000 national health and nutrition examination survey. Diabetes Care 2004; 27: 1591-1597.

24 Toprak A, Reddy J, Chen W, Srinivasan S, Berenson G. Relation of pulse pressure and arterial stiffness to concentric left ventricular hypertrophy in young men (from the Bogalusa Heart Study). Am J Cardiol 2009; 103: 978-984.

25 Suh SY, Kim EJ, Choi CU, Na JO, Kim SH, Kim HJ, Han SW, Chung SM, Ryu KH, Park CG, Seo HS, Oh DJ. Aortic upper wall tissue Doppler image velocity: relation to aortic elasticity and left ventricular diastolic function. Echocardiography 2009; 26: 1069-1074.

26 Vitarelli A, Giordano M, Germanò G, Pergolini M, Cicconetti P, Tomei F, Sancini A, Battaglia D, Dettori O, Capotosto L, De Cicco V, De Maio M, Vitarelli M, Bruno P. Assessment of ascending aorta wall stiffness in hypertensive patients by tissue Doppler imaging and strain Doppler echocardiography. Heart 2010; 96: 1469-1474. 